\title{
A state redistribution algorithm for finite volume schemes on cut cell meshes
}

\author{
Marsha Berger* Andrew Giuliani *
}

\begin{abstract}
In this paper we develop a new technique, called state redistribution, that allows the use of explicit time stepping when approximating solutions to hyperbolic conservation laws on embedded boundary grids. State redistribution is a postprocessing technique applied after each time step or stage of the base finite volume scheme, using a time step that is proportional to the volume of the full cells. The idea is to stabilize the cut cells by temporarily merging them into larger, possibly overlapping neighborhoods, then replacing the cut cell values with a stabilized value that maintains conservation and accuracy. We present examples of state redistribution using two base schemes: MUSCL and a second order Method of Lines finite volume scheme. State redistribution is used to compute solutions to several standard test problems in gas dynamics on cut cell meshes, with both smooth and discontinuous solutions. We show that our method does not reduce the accuracy of the base scheme and that it successfully captures shocks in a non-oscillatory manner.
\end{abstract}

Keywords: cut cells; small cell problem; embedded boundary finite volume scheme

\section{Introduction}

Cut cell meshes to solve hyperbolic problems are increasingly prevalent due to the ease of grid generation for complicated geometries. However, these meshes lead to the small cell problem, which is still an active area of research, and a completely satisfactory solution has not yet been found. The small cell problem can be explained as follows: explicit

${ }^{*}$ Courant Institute, New York University, 251 Mercer St., NY, NY 10012 
finite volume schemes for hyperbolic problems are subject to a CFL constraint, i.e., they typically need to take a time step that is proportional to the mesh width for stability. However, cut cells can have volumes that are arbitrarily smaller than the regular cells. This would force the scheme to take overly restrictive time steps, even though the domain is predominantly composed of regular cells that would otherwise determine the stable time step. Special algorithms are needed to prevent this restriction.

The most commonly used stabilization algorithm is called flux redistribution [6, 10]. The main idea is illustrated below in two space dimensions for ease of notation. The update of the solution average $U_{i, j}^{n}$ on cut cell $(i, j)$ is

$$
V_{i, j} U_{i, j}^{n+1}=V_{i, j} U_{i, j}^{n}-\Delta t \sum_{k \in \text { faces }} A_{k} \mathbf{F}_{k}^{*} \cdot \mathbf{n}_{k}
$$

Here, $\Delta t$ is the time step, volume $V_{i, j}$ is the cell volume, $A_{k}, \mathbf{F}_{k}^{*}$, and $\mathbf{n}_{k}$ are the area, the numerical flux, and the outward facing normal on the $k$ th face, respectively. The above can also be written

$$
V_{i, j} U_{i, j}^{n+1}=V_{i, j} U_{i, j}^{n}+\delta M_{i, j},
$$

where $\delta M_{i, j}$ is the change in the conserved quantity on cell $(i, j)$ after one time step. Instead of using the entire amount of the update in cell $(i, j)$, the cut cell only receives a fraction $\eta_{i, j}$ of it. If the fraction $\eta_{i, j}$ is proportional to the cell's volume fraction $V_{i, j} / V_{\text {full }}$, where $V_{\text {full }}$ is the volume of an uncut Cartesian cell, the update should be stable. To maintain conservation, the rest of the update $\left(1-\eta_{i, j}\right) \delta M_{i, j}$ is given to the cell's neighbors. Flux redistribution has already been implemented for three dimensional calculations due to its simplicity. However it is only first order accurate at the cut cells.

Cell merging [15, 7] is most frequently the first solution that comes to mind for cut cell stabilization. A cut cell is merged with neighbors until a cell with sufficiently large volume for a stable time step is obtained. It is conceptually simple, but we are not aware of any production codes that implement this in a fully general, robust manner for complicated engineering geometries. The $h$-box method [13, 4] is a second order accurate method at the cut cells. It extends the domain of dependence for the fluxes around a small cell in a special way that maintains stability by means of a cancellation property. It has not been extended to three dimensions due to its complexity.

A newer variation of cell merging is called cell linking [5, 17, 14, 7]. This has simpler data structures and maintains the original grid. In [20], the authors improve the accuracy of cell linking, with a third order accurate approach for viscous flow, and fourth order for inviscid flow. Their version of cell linking uses a cluster of cells, while still maintaining each cell in the mesh. A high order polynomial is fit to the cluster, and 
replaces the solution values in the individual cells. Our state redistribution algorithm has a similar spirit to this, though the details are very different.

Two other approaches in the literature include the use of an implicit scheme on the cut cells combined with an explicit scheme elsewhere [19], and a novel flux interpolation scheme which has the added advantage of being dimensionally split, so easier to implement [12]. Our new approach is rather different from these, however, and we do not pursue these directions further.

In this paper we propose a stabilization algorithm in the spirit of flux redistribution. Similar to flux redistribution, state redistribution is applied as a postprocessing step and is simple to implement, for the second order accurate case. We perform an unstable update on all cells with a fixed $\Delta t$ using a base finite volume scheme, followed by a postprocessing step based on the conserved state variables, not on the fluxes. It is for this reason that we call it state redistribution (SRD). Our approach is fully conservative and can be generalized to high order accuracy, albeit with more complexity. The key insight over cell merging was to recognize that we could maintain both conservation and accuracy using a weighted convex combination of solution values that takes into account the number of overlapping neighborhoods on each cell in the base cut cell grid.

The important difference between state redistribution and cell mering is that SRD supports overlapping neighborhoods, and cell merging does not. As a result, cell merging can be difficult to implement robustly in three dimensions, since there are many different, possibly incompatible ways to create non-overlapping merged cells. State redistribution does not suffer from this difficulty, and its extension to three dimensions is straightforward.

The next section illustrates state redistribution in one space dimension on a model problem, to give a more intuitive idea without all the details and notation of the second

order accurate case. Section 3 discusses the evolution schemes on cut cell meshes to which SRD is applied. Section 4 describes the second order accurate algorithm in two space dimension. Computational experiments with the two-dimensional Euler equations are presented in Section 5, and conclusions in Section 6. We see no reason that this algorithm cannot be extended to higher order accuracy, and have already started implementing the third and fourth order accurate cases.

\section{State redistribution in one dimension}

We begin this section by reminding the reader that cell merging can be written as a postprocessing step. We then show that the extension of cell merging for overlapping 
cells does not maintain conservation. This motivates our state redistribution approach, which we contrast with cell merging on a simple one dimensional example. Although the small cells do not mimic the cut cells at the boundary in higher dimensions, this is still a useful model problem.

For the examples in this section we will solve the linear advection equation

$$
u_{t}+a u_{x}=0, \quad a>0
$$

on the nonuniform grid, called the base grid. Equation (1) is discretized using the first order accurate upwind scheme

$$
\widehat{U}_{i}=U_{i}^{n}-\frac{a \Delta t}{h_{i}}\left(U_{i}^{n}-U_{i-1}^{n}\right),
$$

where $U_{i}^{n}$ is the solution average on cell $i$ at time $t^{n}$, the time step $\Delta t$ is constant for all cells. On full cells $h_{i}=h$, and on the small cells $h_{i}=\alpha h$ for $0<\alpha<1$. We first use the grid in Figure 1(a) with one small cell at $i=0$, then the grid in Figure 1(b) with two small cells at $i=-1$ and 1 .

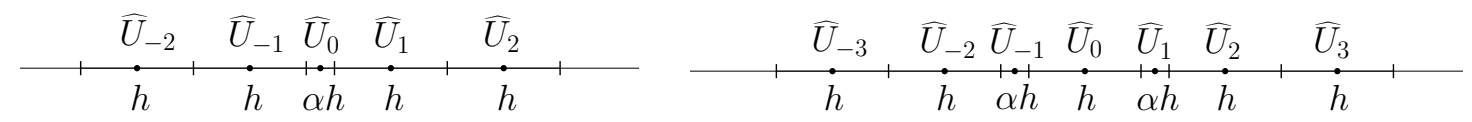

(a)

Figure 1: Model problem in one space dimension on nonuniform base grid. On the left there is one small cell at $i=0$. The right grid has two small cells at $i=-1$ and 1 . The small and large cell sizes are $\alpha h(0<\alpha<1)$, and $h$, respectively.

On the grid in Figure 1(a), cell merging might group cells -1,0, and 1 together into a larger, merged cell. After the unstable update in (2), the volume-weighted merged cell average $\widehat{Q}_{0}$ is computed:

$$
\widehat{Q}_{0}=\frac{\widehat{U}_{-1}+\alpha \widehat{U}_{0}+\widehat{U}_{1}}{2+\alpha} .
$$

The cells comprising the merged cell are then replaced by $\widehat{Q}_{0}$ :

$$
\widehat{U}_{-1}^{n+1}=\widehat{U}_{0}^{n+1}=\widehat{U}_{1}^{n+1}=\widehat{Q}_{0}
$$

This is easily seen to be conservative by checking that

$$
\underbrace{h\left(U_{-1}^{n+1}+\alpha U_{0}^{n+1}+U_{1}^{n+1}\right)}_{\text {mass after redistribution }}=(2+\alpha) h \widehat{Q}_{0}=\underbrace{h\left(\widehat{U}_{-1}+\alpha \widehat{U}_{0}+\widehat{U}_{1}\right)}_{\text {mass before redistribution }}
$$


which equals the values at time $t^{n}$ except for the mass entering and leaving this region.

Next, consider the more complicated case of Figure 1(b). Five cells (indexed by -3 , $-2,0,2,3)$ are large with size $h$ and the remaining two cells (indexed by -1 and 1 ) are small with size $\alpha h$. A first approach to cell merging on this grid might be to make two merged cell averages, $\widehat{Q}_{-1}$ comprising cells $-2,-1,0$, and $\widehat{Q}_{1}$ comprising cells 0,1 and 2 , associated respectively with the small cells -1 and 1 . The first order version here would assign cells as before, except that since cell 0 belongs to two neighborhoods, it seems reasonable to assign $U_{0}^{n+1}=\frac{1}{2}\left(\widehat{Q}_{-1}+\widehat{Q}_{1}\right)$. To check for conservation, we again compute the sum

$$
\begin{array}{r}
\underbrace{h\left(U_{-2}^{n+1}+\alpha U_{-1}^{n+1}+U_{0}^{n+1}+\alpha U_{1}^{n+1}+U_{2}^{n+1}\right)}_{\text {mass after redistribution }}= \\
h\left[(1+\alpha+1 / 2) \widehat{Q}_{-1}+(1 / 2+\alpha+1) \widehat{Q}_{1}\right]= \\
h(3 / 2+\alpha)\left[\frac{\left(\widehat{U}_{-2}+\alpha \widehat{U}_{-1}+\widehat{U}_{0}\right)}{2+\alpha}+\frac{\left(\widehat{U}_{0}+\alpha \widehat{U}_{1}+\widehat{U}_{2}\right)}{2+\alpha}\right] \neq \\
\underbrace{h\left(\widehat{U}_{-2}+\alpha \widehat{U}_{-1}+\widehat{U}_{0}+\alpha \widehat{U}_{1}+\widehat{U}_{2}\right)}_{\text {mass before redistribution }} .
\end{array}
$$

So at least this extension of cell merging to overlapping cells is not conservative.

This motivates the state redistribution procedure, which allows for overlapping cells and stabilizes $\widehat{U}_{i}$ in a conservative manner. This is done by temporarily merging cells of the grid into larger, possibly overlapping, neighborhoods using a specially weighted convex combination, and recombining these averages back onto the grid in a particular fashion. These merged cells are constructed once during a mesh preprocessing step before time stepping.

\section{State redistribution preprocessing}

Each cell in the base grid (both large and small) has a merging neighborhood associated with it. This is a set of neighboring cells with which to temporarily merge. Neighborhoods share the same index as the cell that generated it. Small cells merge with their neighbors until the volume of the merging neighborhood is greater than a threshold, taken here to be half the large cell size of $h / 2$. This is illustrated by the red arrows in Figure 2 where the merging neighborhood of small cell -1 consists of cells $-2,-1$, and 0 , and the merging neighborhood of small cell 1 consists of cells 0,1 , and 2 . Note 


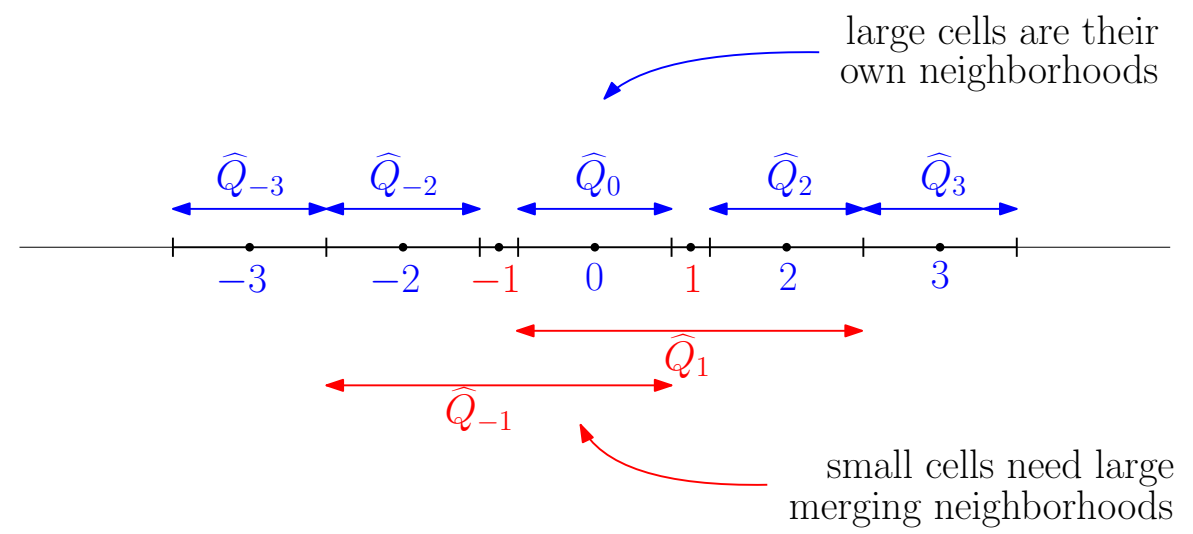

Figure 2: The blue arrows indicate the merging neighborhoods associated with the large cells $-3,-2,0,2$, and 3 , which are their own neighborhoods. The red arrows indicate the neighborhoods of small cells -1 and 1 , which have temporarily merged with their left and right neighbors.

that both neighborhoods overlap on cell 0. Allowing for overlaps makes this temporary merging process simpler. A large cell does not need to merge with neighbors (since $h>h / 2$ ), thus its merging neighborhood is only composed of itself. This is illustrated by the blue arrows in Figure 2. For example, the merging neighborhood of large cell -3 is composed only of itself.

Finally, each cell in the base grid counts the number of neighborhoods that overlap it. Cell -2 is overlapped by two neighborhoods, indexed by -1 and -2 . Cell 0 has 3 such neighborhoods, its own, and one from each small cell adjacent to it.

\section{State redistribution postprocessing}

Using the above information we can now stabilize (2) using the state redistribution method on the grid in Figure $1 \mathrm{~b}$. On each merging neighborhood, we compute a weighted solution average $\widehat{Q}_{i}$, where $i$ is the index of the merging neighborhood. $\widehat{Q}_{i}$ is computed by a convex combination of the averages $\widehat{U}_{i}$ of cells contained in the merging neighborhood, weighted by the inverse of its overlap count. For example, on merging neighborhood -1 , the weighted solution average is

$$
\widehat{Q}_{-1}=\underbrace{\frac{1}{h / 2+\alpha h+h / 3}}_{\text {weighted volume }}(\underbrace{\frac{h}{2} \widehat{U}_{-2}+\alpha h \widehat{U}_{-1}+\frac{h}{3} \widehat{U}_{0}}_{\text {weighted mass }}) .
$$


In formula (7) for the weighted mass, the cell volume is divided by the number of neighborhoods that overlap the associated cell in the base grid. For example, the multiplier in front of $\widehat{U}_{-2}$ is $\frac{h}{2}$ since there are two neighborhoods (from cells -1 and -2 ) that overlap cell -2 . Similarly, the multiplier in front of $\widehat{U}_{-1}$ is $\alpha h$ since there is only one neighborhood (its own) that overlaps cell -1 . Finally, the multiplier in front of $\widehat{U}_{0}$ is $\frac{h}{3}$ since there are three neighborhoods that overlap cell 0 , i.e., cell 0 is overlapped by neighborhoods $-1,1$, and 0 . These multipliers are then divided by the weighted volume, $(h / 2+\alpha h+h / 3)$. iThe weighted solution average on merging neighborhood 1 is similarly defined as

$$
\widehat{Q}_{1}=\frac{1}{h / 2+\alpha h+h / 3}\left(\frac{h}{2} \widehat{U}_{2}+\alpha h \widehat{U}_{1}+\frac{h}{3} \widehat{U}_{0}\right) .
$$

The weighted solution averages on merging neighborhoods that contain only one cell are simply

$$
\widehat{Q}_{i}=\widehat{U}_{i} \quad \text { for } i=-3,-2,0,2,3 \text {. }
$$

The stabilized solution average at time $t^{n+1}$ on a cell in the base grid is then given by the average of all the weighted neighborhood averages that overlap it. On the cell overlapped by three neighborhoods we have

$$
U_{0}^{n+1}=\frac{1}{3}\left(\widehat{Q}_{-1}+\widehat{Q}_{0}+\widehat{Q}_{1}\right)
$$

On the cells overlapped by two neighborhoods, we have

$$
U_{-2}^{n+1}=\frac{1}{2}\left(\widehat{Q}_{-1}+\widehat{Q}_{-2}\right) \text { and } U_{2}^{n+1}=\frac{1}{2}\left(\widehat{Q}_{1}+\widehat{Q}_{2}\right) .
$$

Finally, on cells overlapped by only one neighborhood, we have

$$
U_{i}^{n+1}=\widehat{Q}_{i} \text { for } i=-3,-1,1,3 .
$$

We can write the final solution update on the small cells after SRD at $t^{n+1}$ in terms of the solution averages at $t^{n}$, giving

$$
\begin{aligned}
U_{-1}^{n+1} & =\frac{2-2 \lambda}{5+6 \alpha} U_{0}^{n}+\frac{6 \alpha-4 \lambda}{5+6 \alpha} U_{i-1}^{n}+\frac{3 \lambda+3}{5+6 \alpha} U_{i-2}^{n}+\frac{3 \lambda}{5+6 \alpha} U_{i-3}^{n}, \\
U_{1}^{n+1} & =\frac{2+4 \lambda}{5+6 \alpha} U_{0}^{n}+\frac{6 \alpha-3 \lambda}{5+6 \alpha} U_{i+1}^{n}+\frac{3-3 \lambda}{5+6 \alpha} U_{i+2}^{n}+\frac{2 \lambda}{5+6 \alpha} U_{i-1}^{n} .
\end{aligned}
$$

Before application of the state redistribution method, the weights that multiply the solution averages at time $t^{n}$ in the base scheme (2) become unbounded as $\alpha \rightarrow 0$. 
However, after state redistribution this is no longer the case for the weights in (13). This hints at the stability of our modified scheme.

The state redistribution algorithm allows us to take full time steps as if there were no small cells in the grid. However, it can be seen that the multipliers of $U_{i-1}^{n}$ and $U_{i+1}^{n}$ in $(13)$ are negative when $\alpha$ is small enough. This means that our scheme is not monotone and thus not total variation diminishing. We note that this is also the case with flux redistribution, which has been successfully used in higher dimensions and more complicated problems. The computational examples in Section 5 show that this is not a significant issue for SRD as well. The advantage of state redistribution is that it is linearity preserving, and flux redistribution is not.

\section{Conservation}

We now show that our modified scheme (10), (11), 12) conserves mass. For the portion of the grid in question, the total mass after state redistribution is

$$
\sum_{i} h_{i} U_{i}^{n+1}=h U_{-3}^{n+1}+h U_{-2}^{n+1}+\alpha h U_{-1}^{n+1}+h U_{0}^{n+1}+\alpha h U_{1}^{n+1}+h U_{2}^{n+1}+h U_{3}^{n+1},
$$

where $h_{i}$ is the local cell size. Substituting expressions for the final update (10), (11), (12) into (14), we obtain

$$
\begin{aligned}
\sum_{i} h_{i} U_{i}^{n+1} & =h \widehat{Q}_{-3}+\frac{h}{2}\left(\widehat{Q}_{-1}+\widehat{Q}_{-2}\right) \\
& +\alpha h \widehat{Q}_{-1}+h \frac{1}{3}\left(\widehat{Q}_{-1}+\widehat{Q}_{0}+\widehat{Q}_{1}\right)+\alpha h \widehat{Q}_{1} \\
& +h \frac{1}{2}\left(\widehat{Q}_{1}+\widehat{Q}_{2}\right)+h \widehat{Q}_{3} .
\end{aligned}
$$

Grouping terms in 15 , we have

$$
\begin{aligned}
\sum_{i} h_{i} U_{i}^{n+1} & =h \widehat{Q}_{-3}+\frac{h}{2} \widehat{Q}_{-2} \\
& +\left(\frac{h}{2}+\alpha h+\frac{h}{3}\right) \widehat{Q}_{-1}+\frac{h}{3} \widehat{Q}_{0}+\left(\frac{h}{2}+\alpha h+\frac{h}{3}\right) \widehat{Q}_{1} \\
& +\frac{h}{2} \widehat{Q}_{2}+h \widehat{Q}_{3} .
\end{aligned}
$$


Substituting the expressions for the neighborhood averages (8), (7), (9) into (16), we obtain

$$
\begin{aligned}
\sum_{i} h_{i} U_{i}^{n+1} & =h \widehat{U}_{-3}+\frac{h}{2} \widehat{U}_{-2} \\
& +\left(\frac{h}{2} \widehat{U}_{-2}+\alpha h \widehat{U}_{-1}+\frac{h}{3} \widehat{U}_{0}\right)+\frac{h}{3} \widehat{U}_{0}+\left(\frac{h}{2} \widehat{U}_{2}+\alpha h \widehat{U}_{1}+\frac{h}{3} \widehat{U}_{0}\right) \\
& +\frac{h}{2} \widehat{U}_{2}+h \widehat{U}_{3} .
\end{aligned}
$$

Simplifying (17), the mass after state redistribution becomes

$$
\begin{aligned}
\sum_{i} h_{i} U_{i}^{n+1} & =h \widehat{U}_{-3}+h \widehat{U}_{-2}+\alpha h \widehat{U}_{-1}+h \widehat{U}_{0}+\alpha h \widehat{U}_{1}+h \widehat{U}_{2}+h \widehat{U}_{3} \\
& =\sum_{i} h_{i} \widehat{U}_{i}
\end{aligned}
$$

Thus, the mass on the grid before and after state redistribution does not change. Since the base scheme (2) is conservative, it follows from (18) that our modified scheme (10), (11), 12 is too.

The stabilized finite volume method $(10),(11),(12)$ is first order accurate in space and time. In this work, we provide a framework to generalize the state redistribution method to two dimensional cut cell grids and to second order accuracy in space and time. We will demonstrate with numerical examples that the maximum stable time step is not restricted by the small cells, and that the state redistribution method is conservative.

\section{Second-Order Accurate Base Schemes}

We are interested in solving hyperbolic conservation laws

$$
\frac{\partial}{\partial t} \mathbf{u}+\nabla \cdot \mathcal{F}(\mathbf{u})=\mathbf{0}
$$

on the domain $\Omega \subset \mathbb{R}^{2}$ where $\mathbf{u}(x, y, t) \in \Omega \times[0, T]$ is a vector of conserved quantities, $T$ is the final time, and $\mathcal{F}=[\mathbf{F}, \mathbf{G}]$ is the flux function. We discretize $\Omega$ into a cut cell mesh of cells $\Omega_{i, j}$. A typical cut cell mesh, called the base grid, is given in Figure 3. On the domain interior, $\Omega_{i, j}$ are Cartesian cells (quadrilaterals) of size $\Delta x$ in the $x$ direction and $\Delta y$ in the $y$ direction. On the domain boundary there is a border of 


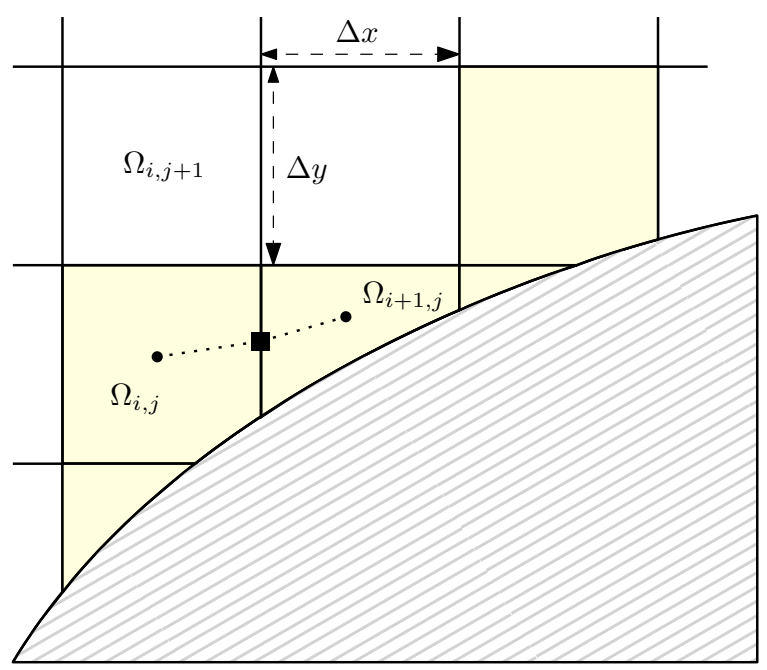

Figure 3: Example base grid in two space dimensions. The cells shaded in yellow are the cut cells. Both the method of lines (Section 3.1) and MUSCL (Section 3.2) schemes require gradient information to reconstruct to the edge midpoint, indicated with a square $(\mathbf{\square})$.

irregular polygonal cells, called cut cells. We use cell-centered discretizations in this work.

There are two issues when applying an explicit finite volume scheme to a cut cell mesh. For accuracy, the scheme needs to be modified in the cut cells. Second, the scheme needs to be stabilized in the cut cells if using a fixed timestep $\Delta t$ based on the full cells. In the $h$-box method these two concerns were addressed simultaneously, but in general they aren't.

We will use two very different second order discretizations of (19): the method of lines (MOL) approach and the MUSCL scheme. These fully discrete finite volume methods, described in Section 3.1 and 3.2, are referred to as the base schemes. Both schemes require linear reconstruction on grid cells, outlined in Section 3.3. Finally, both second order schemes evaluate the flux at the boundary in the examples of Section 5 by extrapolating the pressure to the boundary midpoint. We do not discuss domain boundary conditions in this paper, since these procedures are standard, and do not change due to cut cells. 


\subsection{Method of lines}

After generating the cut cell mesh, we approximate the solution to (19) on the base grid using a finite volume method of the form

$$
\frac{d}{d t} \mathbf{U}_{i, j}=-\frac{1}{V_{i, j}} \int_{\partial \Omega_{i, j}} \mathcal{F}^{*} \cdot \mathbf{n} d l,
$$

where $\mathbf{U}_{i, j}$ is a vector of the cell averages on $\Omega_{i, j}, \partial \Omega_{i, j}$ is the cell boundary, $\mathbf{n}$ is an outward facing normal, and $\mathcal{F}^{*}$ is a numerical flux function.

Second order accuracy in space is achieved by reconstructing a gradient on each cell and using it to evaluate the numerical flux at the face midpoints, illustrated in Figure 3. In our numerical experiments, we use the local Lax-Friedrichs numerical flux to solve the Riemann problem at cell interfaces. The integral in $(20)$ is approximated using the midpoint rule.

Second order accuracy in time is obtained by integrating (20) using Heun's method. This is a two-stage Runge Kutta method that can be written

$$
\begin{aligned}
\mathbf{U}^{(1)} & =\mathbf{U}^{n}+\Delta t L\left(\mathbf{U}^{n}\right), \\
\mathbf{U}^{(2)} & =\mathbf{U}^{(1)}+\Delta t L\left(\mathbf{U}^{(1)}\right), \\
\mathbf{U}^{n+1} & =\frac{1}{2}\left(\mathbf{U}^{n}+\mathbf{U}^{(2)}\right),
\end{aligned}
$$

where $\mathbf{U}^{n}$ is the vector of solution averages on the entire cut cell mesh at time $t^{n}$, $\mathbf{U}^{(1)}, \mathbf{U}^{(2)}$, are intermediate stages, and $L$ is the operator that results from discretizing the right-hand-side of (20). We derive the maximum stable time step on the base grid by using (21) to solve the linear advection equation on a Cartesian grid with advection velocity $(a, b)$. Numerical evaluation of the amplification factor that results from a linear stability analysis reveals that a stable time step satisfies

$$
\Delta t\left(\frac{|a|}{\Delta x}+\frac{|b|}{\Delta y}\right) \leq 1 .
$$

For a discrete maximum principle to be satisfied, a tighter time step restriction

$$
\Delta t\left(\frac{2 \Delta x+2 \Delta y}{\Delta x \Delta y}\right) \sqrt{a^{2}+b^{2}} \leq 1
$$

is required [11], in addition to a limiter to prevent new extrema. For the Euler equations, the advective speeds $a$ and $b$ are replaced by the largest magnitude $x$ and $y$ velocities plus sound speed. 
For this method, we apply SRD stabilization to the stage updates $U^{(1)}$ and $U^{(2)}$ in (21), before they are added to form $U^{n+1}$. This is needed since the tiniest cut cells may have non-physical quantities that need to be stabilized before the next stage. For example, the first step in approximating the Euler equations is to convert the conserved variables to primitives variables. This would break down for negative densities. SRD however does not break down and conservatively and accurately adjusts these values.

\subsection{MUSCL scheme}

The MUSCL scheme is a one step method that is second order accurate in space and time. A series of MUSCL schemes was originated by van Leer [22]. The version we use ${ }^{1}$ is due to Colella [9]. The method is briefly sketched here so that we can describe how it was adapted for cut cells.

On a regular cell $(i, j)$ the interface values on the faces are computed at the half-step in time $t^{n+1 / 2}$, and the left and right states are passed to a Riemann solver to compute the fluxes. Using a Taylor series in space and time to second order, and using the conservation law (19) to replace the derivative in time, gives the value for the right cell interface $(i+1 / 2, j)$

$$
\begin{aligned}
\mathbf{U}_{i+1 / 2, j}^{n+1 / 2} & =\mathbf{U}_{i, j}^{n}+\frac{\Delta t}{2} \frac{\partial \mathbf{U}_{i, j}^{n}}{\partial t}+\frac{\Delta x}{2} \frac{\partial \mathbf{U}_{i, j}^{n}}{\partial x} \\
& =\mathbf{U}_{i, j}^{n}-\frac{\Delta t}{2} \frac{\partial \mathbf{G}_{i, j}^{n}}{\partial y}-\left(\frac{\Delta t}{2} \frac{\partial \mathbf{F}_{i, j}^{n}}{\partial \mathbf{U}_{i, j}^{n}}-\frac{\Delta x}{2}\right) \frac{\partial \mathbf{U}_{i, j}^{n}}{\partial x}
\end{aligned}
$$

The values at the other interfaces of full cells are similarly defined.

In the original method, Riemann problems are solved in the transverse direction, e.g. between centroids $(i, j)$ and $(i, j-1)$, at the edge $j-1 / 2$, to produce $\mathbf{G}_{i, j-1 / 2}$. The terms were then differenced to compute

$$
\partial \mathbf{G}_{i, j} / \partial y=\left(\mathbf{G}_{i, j+1 / 2}-\mathbf{G}_{i, j-1 / 2}\right) / \Delta y .
$$

On a uniform mesh the first order accurate errors in computing 25) cancel, and the term itself is multiplied by $\Delta t$ in (24a). For more details the reader is referred to [9].

At the cut cells the above procedure is no longer accurate, since the centroid values are not coordinate aligned near the cut cells. We make two modifications to the

\footnotetext{
${ }^{1}$ Thanks to Phil Colella for the original Cartesian mesh code and Riemann solver, and for helpful discussions on the shear layer instability and artificial viscosity.
} 
computation of $\partial \mathbf{G} / \partial y$, known as the transverse derivative since it is in the vertical direction when computing the flux $\mathbf{F}$ in the horizontal direction. First, the solution is reconstructed in the transverse direction to the edge midpoint so it is properly centered in cells that are adjacent to a cut cell. This is the situation in Figure 3 for cell $(i, j+1)$, for example.

Second, many cut cells will not have both edges in the transverse $(y)$ direction. Instead, for all cut cells we instead compute $\partial \mathbf{G}_{i, j}^{n} / \partial y=\left(\partial \mathbf{G}_{i, j}^{n} / \partial \mathbf{U}_{i, j}^{n}\right)\left(\partial \mathbf{U}_{i, j}^{n} / \partial y\right)$, like the horizontal fluxes in (24a). This is linearly exact in the cut cells, if the gradients themselves are. We also experimented with dropping this term in the cut cells altogether. There was no stability problem with this, but it does introduce an unnecessary difference from the interior scheme, and would not be linearly exact.

Note: it is the transverse derivative term that provides the so-called corner coupling, i.e. inclusion of corner cells in the stencil. This is what gives the MUSCL scheme a linear stability limit of

$$
\Delta t \max \left(\frac{|a|}{\Delta x}, \frac{|b|}{\Delta y}\right) \leq 1
$$

where $(a, b)$ is the advection velocity.

The trickiest term to adapt to cut cells was an artificial viscosity in the original method that was added to each flux, with a coefficient proportional to the negative divergence of the flow. The original code used a large stencil to compute this divergence. We instead use a centered difference to compute $u_{x}$ and $v_{y}$, and where possible, and take the max of this over a $3 \times 3$ neighborhood centered around each cell, so that cut cells get this dissipation too.

The multi-dimensional MUSCL scheme due to Colella has several additional features to robustly handle strong shocks, such as not including terms in predicting the interface state from characteristics that propagate away from the interface. These steps do not change at the cut cells, so are not discussed here.

Since MUSCL is a one-step scheme, the SRD stabilization is applied directly before the final update $\mathbf{U}^{n+1}=S R D\left(\mathbf{U}^{n}+\Delta t L\left(\mathbf{U}^{n}\right)\right)$, where $L$ is now the MUSCL operator.

\subsection{Gradient reconstruction and limiting}

The computation of gradients, and for problems with discontinuities limiting those gradients, arises independently of the finite volume scheme used. On the domain interior, when the stencil is regular and does not contain cut cells, standard schemes can be used. In all our examples we use monotonized central (MC) differencing in both $x$ and 
$y$ directions. The MC limited slope in the $x$ direction is

$$
\sigma_{x, i, j}^{n}=\left\{\begin{array}{lc}
\min \left(\left|D_{c}\right|, 2\left|D_{+}\right|, 2\left|D_{-}\right|\right) \times \operatorname{sign} D_{c}, & \text { if } D_{+} D_{-}>0 \\
0 & \text { otherwise }
\end{array}\right.
$$

Here we use the standard differencing notation $D_{c}=\left(U_{i+1, j}^{n}-U_{i-1, j}^{n}\right) /(2 \Delta x)$ for the second order accurate central difference and $D_{+}=\left(U_{i+1, j}^{n}-U_{i, j}^{n}\right) / \Delta x, D_{-}=\left(U_{i, j}^{n}-\right.$ $\left.U_{i-1, j}^{n}\right) / \Delta x$ for the one-sided differences. The MC limited slope in the $y$ direction is similarly defined.

For cut cells we use a least squares gradient reconstruction algorithm, a standard procedure for unstructured meshes. A linear reconstruction of the solution on these cells is of the form

$$
u_{i, j}^{n}(x, y)=U_{i, j}^{n}+\sigma_{x, i, j}^{n}\left(x-x_{i, j}\right)+\sigma_{y, i, j}^{n}\left(y-y_{i, j}\right),
$$

where $(i, j)$ is the index of either a cut cell or cell with an irregular stencil, $\left(\sigma_{x}^{n}, \sigma_{y}^{n}\right)_{i, j}$ and $(x, y)_{i, j}$ are its gradient and cell centroid, respectively. The least squares procedure finds the gradient that minimizes the $L_{2}$ residual when evaluating $u_{i, j}^{n}(x, y)$ at neighboring cell centroids.

In this work, we consider both first and second order accurate gradients. The reconstructed first order gradient satisfies in the least squares sense

$$
\sigma_{x, i, j}^{n}\left(x_{r, s}-x_{i, j}\right)+\sigma_{y, i, j}^{n}\left(y_{r, s}-y_{i, j}\right)=U_{r, s}^{n}-U_{i, j}^{n} \quad \forall(r, s) \in R_{i, j},
$$

where $R_{i, j}$ is the set of cell indices used for slope reconstruction on cell $(i, j)$ in the base scheme. Here, $R_{i, j}$ is the $3 \times 3$ neighborhood centered on $(i, j)$. The reconstructed second order gradient satisfies in the least squares sense

$$
\begin{aligned}
& \sigma_{x, i, j}^{n}\left(x_{r, s}-x_{i, j}\right)+\sigma_{y, i, j}^{n}\left(y_{r, s}-y_{i, j}\right)+ \\
& \quad \frac{1}{2} \sigma_{x x, i, j}^{n}\left[\left(x_{r, s}-x_{i, j}\right)^{2}-S_{x x, i, j}\right]+ \\
& \quad \sigma_{x y, i, j}^{n}\left[\left(x_{r, s}-x_{i, j}\right)\left(y_{r, s}-y_{i, j}\right)-S_{x y, i, j}\right]+ \\
& \frac{1}{2} \sigma_{y y, i, j}^{n}\left[\left(y_{r, s}-y_{i, j}\right)^{2}-S_{y y, i, j}\right]=U_{r, s}^{n}-U_{i, j}^{n} \quad \forall(r, s) \in R_{i, j},
\end{aligned}
$$

where $\sigma_{x x, i, j}^{n}, \sigma_{x y, i, j}^{n}, \sigma_{y y, i, j}^{n}$ are quadratic degrees of freedom and are discarded. In this case, $R_{i, j}$ is either the $3 \times 3$ tile centered on $(i, j)$ when $(i, j)$ is a whole cell neighboring a cut cell, or the $5 \times 5$ tile centered on $(i, j)$ when $(i, j)$ is a cut cell. Note that a cut cell needs a larger neighborhood because approximately half of its cells are not in the flow domain. 
Regular cells that are adjacent to a cut cell will also need special treatment to compute a second order accurate gradient. We have experimented with three approaches and found almost indistinguishable results. The simplest is to use the procedure mentioned above for cut cells - fit a least squares polynomial in the $3 \times 3$ neighborhood centered on that cell. We also tried $5 \times 5$ neighborhoods, in hope of smoother transitions between cut cells and the interior cells. Finally, we tried using a centered gradient in only one dimension, if there was one, and using a recentering approach to compute the second order accurate difference in the other direction. This has an overall smaller stencil, but still uses the $3 \times 3$ neighborhood if there is no regular direction, and involves more testing.

For problems with discontinuities, the gradient will need to be limited to prevent overshoots and retain positivity for quantities like density and pressure. We use the Barth Jespersen (BJ) limiter 2 to limit on cut cell grids. This is a scalar limiter, where both $\sigma_{x, i, j}^{n}$ and $\sigma_{y, i, j}^{n}$ are reduced by the same scalar to prevent new extrema. We compute the minimum and maximum values over the reconstruction stencil $R_{i, j}$,

$$
m_{i, j}=\max _{(r, s) \in R_{i, j}} U_{r, s}^{n} \text { and } M_{i, j}=\max _{(r, s) \in R_{i, j}} U_{r, s}^{n} .
$$

The reconstructed gradient on cell $(i, j)$ is limited by a non-negative scalar $\alpha \in[0,1]$, so that when $u_{i, j}(x, y)$ is evaluated at the centroids of the neighborhoods in $R_{i, j}$ it lies between $m_{i, j}$ and $M_{i, j}$. (Apologies for reusing the symbol $\alpha$, since it is commonly used to describe Barth-Jespersen-type limiters, as well as the mesh width $\alpha h$ of small cells).

The limited numerical solution is

$$
\tilde{u}_{i, j}^{n}(x, y)=U_{i, j}^{n}+\alpha\left[\sigma_{x, i, j}^{n}\left(x-x_{i, j}\right)+\sigma_{y, i, j}^{n}\left(y-y_{i, j}\right)\right] .
$$

Define

$$
\alpha_{r, s}= \begin{cases}\min \left(1, \frac{M_{i, j}-U_{i, j}^{n}}{U_{r, s}^{n}-U_{i, j}^{n}}\right) & \text { if } U_{r, s}^{n}-U_{i, j}^{n}>0 \\ \min \left(1, \frac{m_{i, j}-U_{i, j}^{n}}{U_{r, s}^{n}-U_{i, j}^{n}}\right) & \text { if } U_{r, s}^{n}-U_{i, j}^{n}<0 . \\ 1 & \text { if } U_{r, s}^{n}-U_{i, j}^{n}=0 .\end{cases}
$$

Then choose

$$
\alpha=\min _{(r, s) \in R_{i, j}} \alpha_{r, s}
$$

By reconstructing to the neighboring cell centroids, this procedure is linearity preserving.

The approach described above differs slightly from the original Barth-Jespersen limiter in [2], where the numerical solution is reconstructed to points on cell interfaces. 
This is often the procedure used on unstructured meshes. However cut cell meshes are much more irregular and without this fix BJ can lead to much less accurate solutions.

\section{State redistribution in two dimensions}

This section describes the second order accurate state redistribution algorithm. We will show that SRD preserves linear functions and is conservative, since it is not obvious that the unusual weightings in our algorithm preserve these important properties.

\subsection{State redistribution preprocessing}

In this section, we describe mesh dependent quantities that will be used when applying SRD. Each cut cell needs two pieces of information: the cells that belong to its own merging neighborhood, and how many neighborhoods it belongs to. This is the twodimensional analogue of the one-dimensional nonuniform grid preprocessing presented in Section 2, now done on cut cell grids. The preprocessing determines these quantities: merging neighborhoods and overlap counts, weighted volumes and centroids. For moving geometries, they would need to be be recomputed when the geometry is modified.

\section{Merging neighborhoods}

In one dimension, neighborhoods can be defined by merging a small cell with neighbors on its left, on its right, or both. Of these three approaches, the last was used on the nonuniform grid presented in Section 2. In two dimensions, there is much more freedom in defining a merging neighborhood. We investigated two different ways: (1) normal merging and (2) centered merging.

Normal merging associates cut cells with neighbors in the direction closest to the boundary normal. This is illustrated in Figure 4, where the normal merging neighborhood associated with cut cell $(i, j)$ is highlighted in green.

Centered merging associates cut cells with neighbors that are symmetrically located in each direction around the cell. These cells are located in the $3 \times 3$ tile centered on the small cell. Figure 4 illustrates this for cut cell $(i+4, j+1)$, where the centered neighborhood is highlighted in green.

The larger the neighborhood the more diffusive the results. Thus we do not want neighborhoods that are too large. However, they must satisfy a size constraint to 


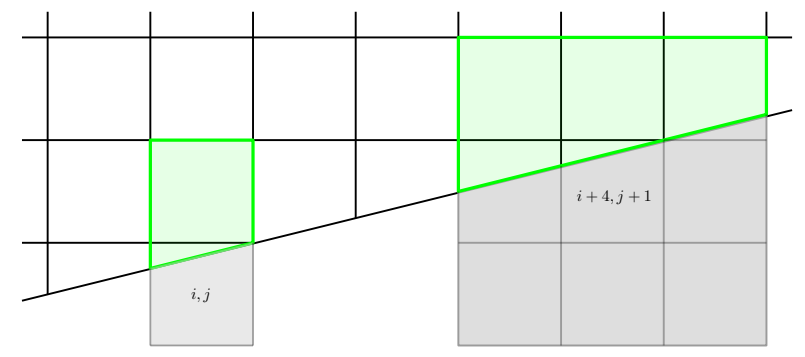

Figure 4: On the left, a small cell is merged with a cell in the direction closest to the boundary normal. On the right, a small cell is merged with neighbors that are at most one cell away, i.e., cells located in the $3 \times 3$ tile centered on $(i+4, j+1)$.

dampen unstable growth in the numerical solution. To this end, we determine neighborhoods, either normal or centered, such that the volume of the neighborhood is at least half the volume of an uncut cell, i.e.,

$$
\sum_{(r, s) \in M_{i, j}} V_{r, s} \geq \frac{1}{2} \Delta x \Delta y,
$$

where $M_{i, j}$ denotes the set of cell indices that belong to merging neighborhood $(i, j)$. In one dimension it has been shown [3] that a cut cell at the boundary that is at least half the regular cell size is stable using a full time step $\Delta t$. We have not encountered any issues with this choice in two dimensions.

For smooth solutions a centered neighborhood is sufficient, but for shocks this yields unsatisfactory results. Therefore, we use the normal neighborhood everywhere possible. The normal neighborhood cannot be used when, e.g., a neighboring cell is also cut and the merging neighborhood is not sufficiently large (Figure 5a). In this case, we must use central merging with cells on a $3 \times 3$ tile (Figure 5b), or, if that merging neighborhood is not large enough, with cells on the $5 \times 5$ tile. In this Figure, the gray cells are exterior to the fluid domain, and are drawn for context.

After forming the merging neighborhoods, each cell counts the number of neighborhoods it belongs to. For cell $(i, j)$, this neighborhood count is called $N_{i, j}$.

To more clearly illustrate overlapping merging neighborhoods in two dimensions, consider the cut cell mesh in Figure 6. The neighborhoods $(i, j)$ and $(i, j+1)$ overlap on cell $(i, j+1)$ in the base grid. The normal merging neighborhood of cut cell $(i, j)$ is highlighted in green in Figure 6a. Since cell $(i, j)$ does not satisfy the volume constraint in (35), the neighborhood of $(i, j), M_{i, j}$, must include both $(i, j)$ and $(i, j+1)$. The neighborhood associated with $(i, j+1)$ in highlighted in green in Figure 6b. Since the 


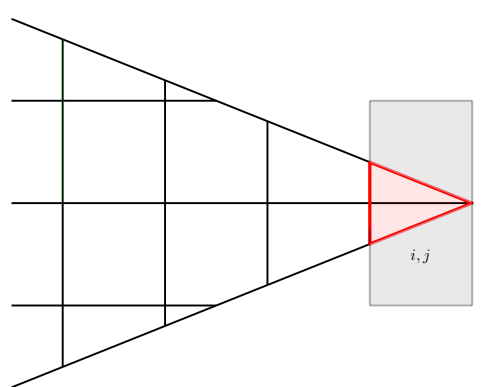

(a) Normal neighborhood (in red) for the cut cell $(i, j)$.

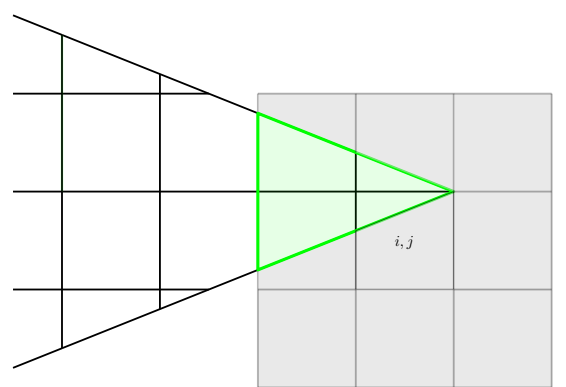

(b) $3 \times 3$ merging neighborhood (in green) for centered on cut cell $(i, j)$.

Figure 5: It can happen that merging only in the normal neighborhood is not large enough, e.g. if the small cell merges with another small cell, and 35 is not satisfied. In this case, we use the $3 \times 3$ neighborhood.

volume of cell $(i, j+1)$ satisfies the volume constraint in $(35), M_{i, j+1}$, only contains cell $(i, j+1)$.

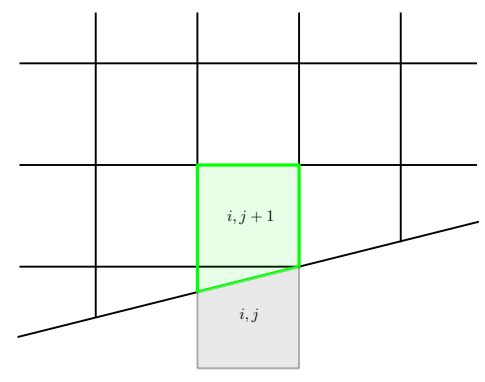

(a) Merging neighborhood of cell $(i, j)$.

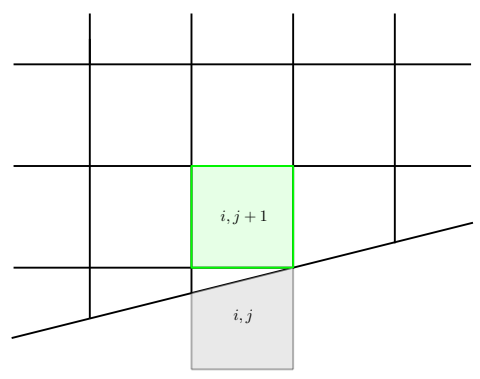

(b) Merging neighborhood of cell $(i, j+1)$.

Figure 6: Example of two neighborhoods, highlighted in green, that overlap on cell $(i, j+1)$.

\section{Weighted centroids and volumes}

The neighborhood's weighted volume is defined as

$$
\widehat{V}_{i, j}=\sum_{(r, s) \in M_{i, j}} \frac{V_{r, s}}{N_{r, s}}
$$


where $M_{i, j}$ is the set of cells in the neighborhoods associated with cell $(i, j)$. The weighted centroid of the merging neighborhood is defined as

$$
\left(\widehat{x}_{i, j}, \widehat{y}_{i, j}\right)=\frac{1}{\widehat{V}_{i, j}} \sum_{(r, s) \in M_{i, j}} \frac{V_{r, s}}{N_{r, s}}\left(x_{r, s}, y_{r, s}\right),
$$

In general, the weighted volume is not the physical volume of the merging neighborhood unless the neighborhood is not overlapped by any other neighborhoods (for example, cells -3 and 3 in Figure 2). Similarly, the weighted and physical centroids are different unless the overlap count on each cell in the neighborhood is the same, $N_{r, s}=N_{p, q} \forall(r, s),(p, q) \in M_{i, j}$. This is because the overlap counts in (37) would cancel, leading to identical weighted and physical centroids of the neighborhood.

\subsection{State redistribution postprocessing}

In this section, we describe postprocessing on two-dimensional meshes with overlapping neighborhoods. This is the analogue of postprocessing on one dimensional nonuniform grids in Section 2. The SRD stabilization is applied after each stage for the method of lines (Section 3.1) or time step for the MUSCL scheme (Section 3.2), denoted generically as

$$
\widehat{U}=U^{n}+\Delta t L\left(U^{n}\right) .
$$

We refer to $\widehat{U}$ as the provisionally updated solution.

\section{Step 1. Compute weighted solution averages on each neighborhood}

The solution average on each neighborhood is given by

$$
\widehat{Q}_{i, j}=\frac{1}{\widehat{V}_{i, j}} \sum_{(r, s) \in M_{i, j}} \frac{V_{r, s}}{N_{r, s}} \widehat{U}_{r, s}
$$

where the volume $\widehat{V}_{i, j}$ is the weighted neighborhood volume defined in (36) and $N_{r, s}$ is the number of neighborhoods associated with cell $i, j$ defined in Section 4.1. Analogous to the one dimensional case in Section 2, the weighted solution averages are a convex combination of provisional solution averages on the neighborhood associated with cell $(i, j)$. 


\section{Step 2. Reconstruct and limit a gradient on each neighborhood}

For second order accuracy in space we need to compute a gradient on each neighborhood, again using a least squares procedure. The set of merging neighborhood indices for reconstruction on the neighborhood associated with cell $(i, j)$ is called $\widehat{R}_{i, j}$. We note that this set need not be the same as $R_{i, j}$ used in the cut cell gradient reconstruction on the base grid (Section 3.3). Similar to the base grid reconstruction in (28), the reconstruction on neighborhood $(i, j)$ is of the form

$$
\widehat{q}_{i, j}(x, y)=\widehat{Q}_{i, j}+\widehat{\sigma}_{x, i, j}\left(x-\widehat{x}_{i, j}\right)+\widehat{\sigma}_{y, i, j}\left(y-\widehat{y}_{i, j}\right),
$$

where $\widehat{Q}_{i, j}$ is the weighted neighborhood average defined in $(39),\left(\widehat{x}_{i, j}, \widehat{y}_{i, j}\right)$ is the weighted centroid of neighborhood $(i, j)$, and $\left(\widehat{\sigma}_{x, i, j}, \widehat{\sigma}_{y, i, j}\right)$ is the gradient on the merging neighborhood. The neighborhood gradient $\left(\widehat{\sigma}_{x, i, j}, \widehat{\sigma}_{y, i, j}\right)$ satisfies in the least squares sense

$$
\widehat{\sigma}_{x, i, j}\left(\widehat{x}_{r, s}-\widehat{x}_{i, j}\right)+\widehat{\sigma}_{y, i, j}\left(\widehat{y}_{r, s}-\widehat{y}_{i, j}\right)=\widehat{Q}_{r, s}-\widehat{Q}_{i, j} \quad \forall(r, s) \in \widehat{R}_{i, j} .
$$

Note that (41) uses the neighborhood's weighted centroids $\left(\widehat{x}_{r, s}, \widehat{y}_{r, s}\right)$ instead of the cell centroids. In Section 4.3, we prove that this procedure is linearity preserving. Alternatively, second order gradients on the neighborhoods can be obtained by fitting a quadratic as in Section 3.3, and discarding the second derivative terms. We will compare these procedures in the computational results in Section 5.1 .

The set of neighborhoods used for gradient reconstruction on neighborhood $(i, j)$, $\widehat{R}_{i, j}$, is the $3 \times 3$ tile. It could happen that this set does not contain enough neighborhoods, or that the weighted centroids of these neighborhoods are too close to compute a well-conditioned gradient. This is the case in Figure 7, where the weighted centroids are too close in the $y$ direction.

We remedy this by increasing the stencil size for the gradient computation if the neighborhood does not contain another weighted centroid at least $\frac{1}{2} \Delta x$ and $\frac{1}{2} \Delta y$ away in the $x$ or $y$ direction respectively. For example, if the weighted centroids are too close in the $x$ direction, but not the $y$ direction, then the $5 \times 3$ tile is used as the reconstruction neighborhood. Similarly, if the weighted centroids are too close in the $y$ direction, but not the $x$ direction, then the $3 \times 5$ tile is used as the reconstruction neighborhood. The neighborhood size is increased until this distance requirement is satisfied in both $x$ and $y$ directions. In Figure 7, the appropriate reconstruction neighborhood is the $3 \times 5$ reconstruction tile.

To limit the reconstructed gradient we apply the BJ limiter, this time over $\widehat{R}_{i, j}$ rather than $R_{i, j}$ in (31) and (34). This procedure is also linearity preserving. 


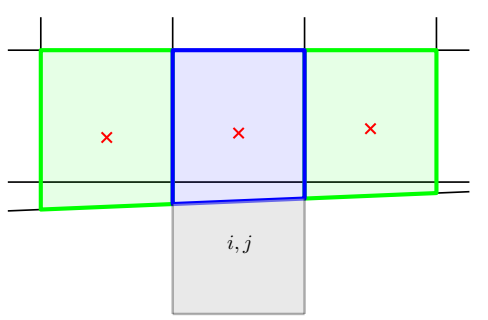

(a) The blue merging neighborhood is associated with cut cell $(i, j)$. The green merging neighborhoods are associated with cut cells $(i-1, j)$ and $(i+1, j)$.

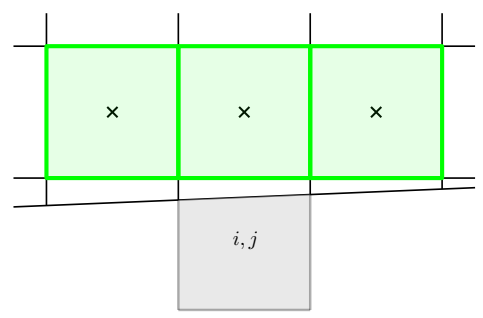

(b) The green merging neighborhoods are associated with the whole cells $(i-1, j+1)$, $(i, j+1)$, and $(i+1, j+1)$.

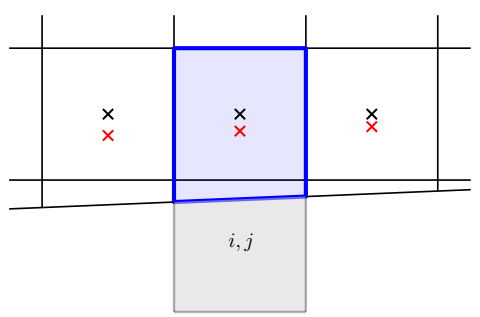

(c) The weighted centroids of the reconstruction neighborhoods in Figures $7 \mathrm{a}, 7 \mathrm{~b}$.

Figure 7: The weighted centroids of the neighborhoods in $\widehat{R}_{i, j}$ are indicated with a cross $(\times)$. Here $\widehat{R}_{i, j}$ is the set of merging neighborhoods associated with cells on the $3 \times 3$ tile centered on cell $(i, j)$. Clearly, the weighted centroids are too close to one another in the $y$ direction (Figure 7c). It follows that least squares system to reconstruct the $x$ and $y$ derivatives of the numerical solution on the blue merging neighborhood is very ill-conditioned.

\section{Step 3. Final solution update}

The final update on cell $(i, j)$ is the average of all its neighborhood reconstructions evaluated at $(i, j)$ 's physical centroid $\left(x_{i, j}, y_{i, j}\right)$. This is given by

$$
U_{i, j}^{n+1}=\frac{1}{N_{i, j}} \sum_{(r, s) \in W_{i, j}} \hat{q}_{r, s}\left(x_{i, j}, y_{i, j}\right) .
$$

where $W_{i, j}$ is the list of indices of the neighborhoods that overlap cell $(i, j)$.

Note: The final update formula 42 can easily be implemented with a nested for loop as in Algorithm 1, instead of computing the set $W_{i, j}$ in 42. The outer loop iterates over the merging neighborhoods $(i, j)$ and the inner loop iterates over each cell $(r, s)$ in neighborhood $(i, j)$. Each merging neighborhood $(i, j)$ gives a contribution $\hat{q}_{i, j}\left(x_{r, s}, y_{r, s}\right) / N_{r, s}$ to the cells $(r, s)$ that belong to it. 


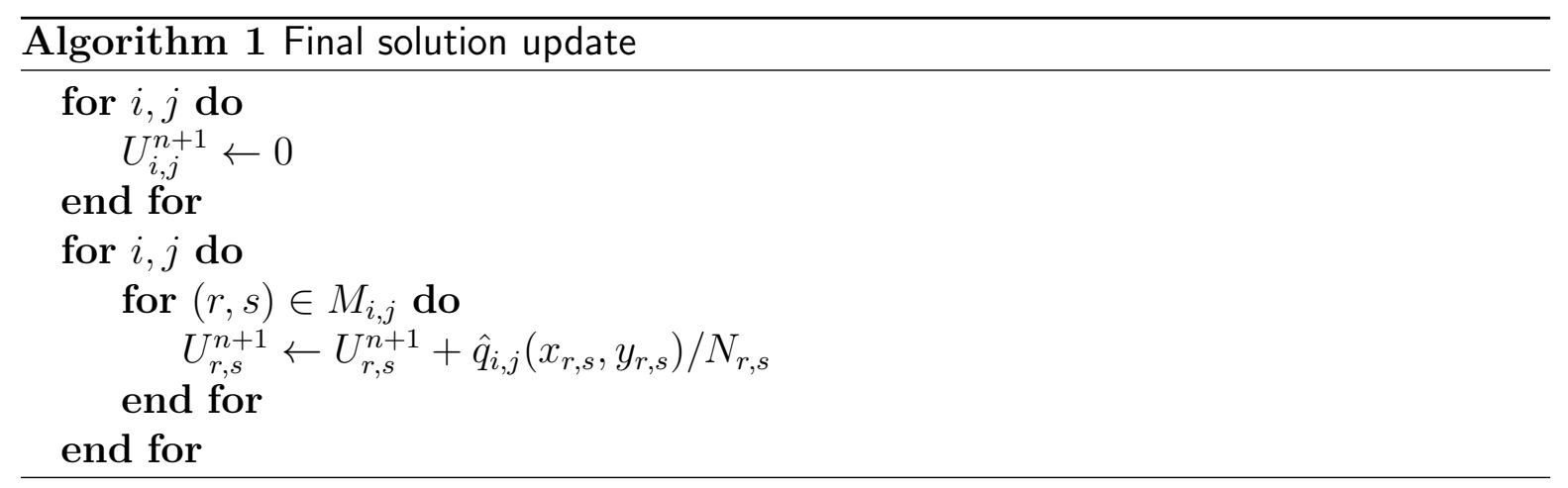

\subsection{Linear exactness}

In this section, we show that second order accurate state redistribution preserves linear functions. One might wonder about this since the centroids and solution averages are weighted in this unusual way. In addition, the local truncation error does not imply the order of accuracy of the scheme as it does on regular meshes [23]. Here we simply show that a linear function remains exact after SRD, if the base scheme is linearly exact.

Consider the grid function of the numerical solution after one time step or stage, $\widehat{U}$. Assume that it can be written in terms of a linear function $f(x, y)$ of the $x$ and $y$ coordinates, i.e.,

$$
\widehat{U}_{i, j}=f\left(x_{i, j}, y_{i, j}\right)=a x_{i, j}+b y_{i, j}+c .
$$

This assumption is valid since both the method of lines (Section 3.1) and the MUSCL scheme (Section 3.2) are linearity preserving. From (43) and the expression for the average on the merging neighborhood $(i, j)$ in $(39)$, we have

$$
\widehat{Q}_{i, j}=\frac{1}{\widehat{V}_{i, j}} \sum_{(r, s) \in M_{i}} \frac{V_{r, s}}{N_{r, s}}\left(a x_{r, s}+b y_{r, s}+c\right) .
$$

Distributing the summation in (44), we have

$\widehat{Q}_{i, j}=a\left(\frac{1}{\widehat{V}_{i, j}} \sum_{(r, s) \in M_{i, j}} \frac{V_{r, s}}{N_{r, s}} x_{r, s}\right)+b\left(\frac{1}{\widehat{V}_{i, j}} \sum_{(r, s) \in M_{i, j}} \frac{V_{r, s}}{N_{r, s}} y_{r, s}\right)+c\left(\frac{1}{\widehat{V}_{i, j}} \sum_{(r, s) \in M_{i, j}} \frac{V_{r, s}}{N_{r, s}}\right)$.

From the definition of the weighted centroid and volume of the merging neighborhood $(i, j)$ in (36) and (37), respectively, (45) becomes

$$
\widehat{Q}_{i, j}=a \widehat{x}_{i, j}+b \widehat{y}_{i, j}+c=f\left(\widehat{x}_{i, j}, \widehat{y}_{i, j}\right) .
$$


Now, on neighborhood $(i, j)$, we solve the least squares system (41) to find $\widehat{\sigma}_{x, i, j}$ and $\widehat{\sigma}_{y, i, j}$, the gradient on the merging neighborhood. Using (46) in (41), and due to the linearity of $f$, the following system

$$
\widehat{\sigma}_{x, i, j}\left(\widehat{x}_{r, s}-\widehat{x}_{i, j}\right)+\widehat{\sigma}_{y, i, j}\left(\widehat{y}_{r, s}-\widehat{y}_{i, j}\right)=f\left(\widehat{x}_{r, s}, \widehat{y}_{r, s}\right)-f\left(\widehat{x}_{i, j}, \widehat{y}_{i, j}\right) \quad \forall(r, s) \in \widehat{R}_{i, j},
$$

is solved exactly by $\widehat{\sigma}_{x, i, j}=a$ and $\widehat{\sigma}_{y, i, j}=b$. In other words, the exact gradient of $f$ is reconstructed on merging neighborhood $(i, j)$. The reconstructed solution is then

$$
\hat{q}_{i, j}(x, y)=f\left(\widehat{x}_{i, j}, \widehat{y}_{i, j}\right)+a\left(x-\widehat{x}_{i, j}\right)+b\left(y-\widehat{y}_{i, j}\right) .
$$

Using (46), (48) becomes

$$
\hat{q}_{i, j}(x, y)=f(x, y) .
$$

By 42 , the final solution update is

$$
U_{i, j}^{n+1}=\frac{1}{N_{i, j}} \sum_{(r, s) \in W_{i, j}} f\left(x_{i, j}, y_{i, j}\right) .
$$

The function values are exact, and there are $N_{i, j}$ of them, so after dividing by $N_{i, j}$ we get

$$
U_{i, j}^{n+1}=f\left(x_{i, j}, y_{i, j}\right),
$$

which shows that second order accurate state redistribution preserves linear functions.

\subsection{Conservation}

In this section, we show that the total mass of the numerical solution before and after

state redistribution does not change. It follows from the final update in (42) that the total mass after state redistribution is

$$
\sum_{i, j} V_{i, j} U_{i, j}^{n+1}=\sum_{i, j} \sum_{(r, s) \in M_{i, j}} \frac{1}{N_{r, s}} V_{r, s} \widehat{q}_{i, j}\left(x_{r, s}, y_{r, s}\right),
$$

where $\widehat{q}_{i, j}(x)$ is that neighborhood's polynomial reconstruction defined in (40). Using the definition of $\widehat{q}_{i, j}(x, y)$, we have

$$
\sum_{i, j} V_{i, j} U_{i, j}^{n+1}=\sum_{i, j} \widehat{Q}_{i, j} \widehat{V}_{i, j}
$$


Using the definition of the weighted average (39), we have

$$
\sum_{i, j} V_{i, j} U_{i, j}^{n+1}=\sum_{i, j} \sum_{(r, s) \in M_{i, j}} \frac{V_{r, s}}{N_{r, s}} \widehat{U}_{r, s} .
$$

Since $N_{r, s}$ indicates the number of times cell $(r, s)$ is overlapped by merging neighborhoods, it follows that the $\frac{V_{r, s}}{N_{r, s}} \widehat{U}_{r, s}$ term is repeated $N_{r, s}$ times in the sum of (54). Thus, simplifying (54), it follows that

$$
\sum_{i, j} V_{i, j} U_{i, j}^{n+1}=\sum_{i, j} V_{i, j} \widehat{U}_{i, j}
$$

and the total mass before and after state redistribution is the same.

\section{Computational Results}

In this section we show several computational experiments using state redistribution to solve the Euler equations. We will also use these examples to examine properties of different gradient choices and base schemes.

\subsection{Supersonic vortex study}

We compute the solution to a supersonic flow around a quarter circle. This problem has often been used in accuracy studies [1] since it has an exact solution to the Euler equations that is smooth, given by

$$
\rho=\rho_{i}\left\{1+\frac{\gamma-1}{2} M_{i}^{2}\left[1-\left(\frac{r_{i}}{r}\right)^{2}\right]\right\}^{\frac{1}{\gamma-1}}
$$

and $u=a_{i} M_{i}\left(\frac{r_{i}}{r}\right) \sin (\theta), v=-a_{i} M_{i}\left(\frac{r_{i}}{r}\right) \cos (\theta)$, and $p=\rho^{\gamma} / \gamma$. Here, the inner radius is $r_{i}=1.0$, the outer radius is $r=1.384, \rho_{i}=1$, and the Mach number on the inner circle $M_{i}=2.25$ in our experiments. In this normalization we use $a_{i}=1, p_{i}=1 / \gamma$. The second order MOL scheme is used, and the time step is chosen using a CFL of 1.0 in (22) based on the regular cell volume.

We march to steady state, until the maximum density update is below $10^{-10}$. The solution is smooth, so no limiters are needed. The exact solution is used to set the ghost cells at the inflow and outflow boundaries. The domain size is $[0,1.43]$ by $[0.0,1.4301]$ (slightly different to prevent mesh degeneracies). The density profile and computational domain is illustrated in Figure 8, 
This example will demonstrate the accuracy of SRD. We also use this example to compare the accuracy of different formulations for the cut cell and SRD neighborhood gradients, since as noted previously, gradients are an important part of this algorithm. Table 1 we compares the accuracy of three such formulations. For first order accurate gradients, we use a linear least squares reconstruction for both the irregular cell gradient (cut cells and their one-away neighbors) described in Section 3.3 , and the SRD gradients, which update the cut cell solution after stabilization. Second order accurate gradients fit a quadratic for both the cut cells and tiles as described in Section 3.3, but only the first derivative terms are used. As an intermediate experiment, we fit a quadratic using least squares but treat the cell

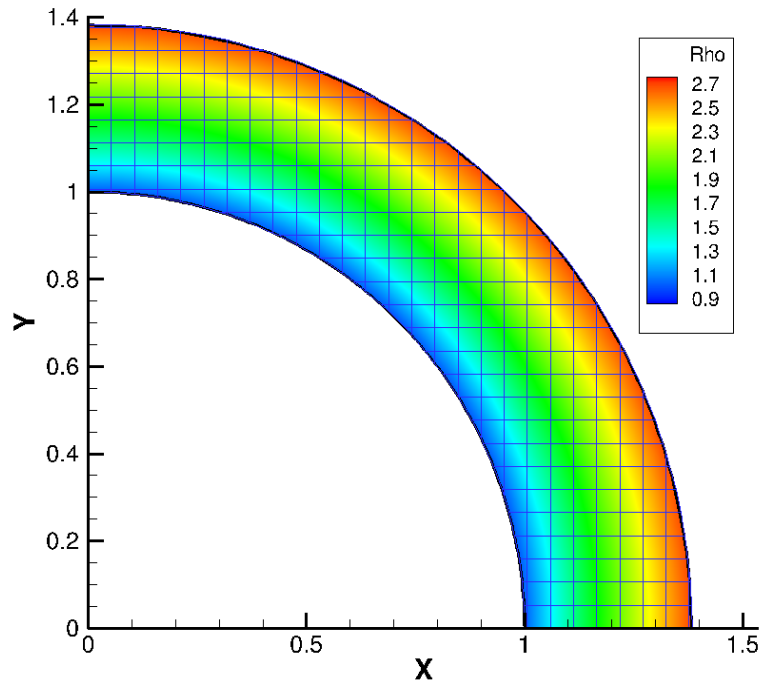

Figure 8: The domain is discretized by 27 cells in each direction, and the exact density profile is shown. Ghost cells and solid cells are not shown. averages as pointwise values at the cell centers. Here again the second derivative terms are ignored. We refer to this as "pointwise quadratic reconstruction". Note that this is not a second order accurate gradient, since the centroid value differs by $O\left(h^{2}\right)$ from the pointwise value at the centroid. Nevertheless, this is frequently done since it is easier to implement. This is particularly true for SRD neighborhoods with irregular shapes.

We measure the $L_{1}$ norm of the error in the volume, $\sum_{i, j} V_{i j}\left|e_{i j}\right|$ and at the boundary, $\sum_{i, j \in \partial \Omega} A_{i j}\left|e_{i j}\right|$. Here $e_{i, j}$ is the error at the cell centroid, and $A_{i j}$ is the length of the boundary segment in cut cell $(i, j)$. These are given in Table $1 \mathrm{a}$ and $1 \mathrm{~b}$. For easier comparison with other papers we also give the mesh size to a few digits. In a cut cell mesh there are both solid and flow cells, so the total number of cells is larger than the number of flow cells, and not as easy to compare as the mesh spacing. All interior cells use the same evolution scheme and gradients, so the difference in the errors is solely due to the irregular cell reconstruction scheme.

Note that the error at the cut cells is larger than in the volume, and has a lower convergence rate. Since the number of cut cells grows only linearly with refinement, the $L_{1}$ accuracy in the entire flow field is still second order. At the boundary, Richardson extrapolation shows that the convergence rate seems to be between 1.38 and 1.5. This has also been found in other cut cell studies [18, 21], and is due to the irregularity of the difference scheme at the cut cells, and the curved boundary. The next example will show 


\begin{tabular}{|l|c|l|l|l|}
\hline$h$ & $N_{x}, N_{y}$ & 1st order grad. & ptwise grad. & 2nd order grad. \\
\hline .5297 & 27 & $6.75 \mathrm{e}-3$ & $2.76 \mathrm{e}-3$ & $2.45 \mathrm{e}-3$ \\
\hline .2648 & 54 & $1.78 \mathrm{e}-3(3.8)$ & $6.38 \mathrm{e}-4(4.3)$ & $4.71 \mathrm{e}-4(5.2)$ \\
\hline .1324 & 108 & $3.63 \mathrm{e}-4(4.9)$ & $1.53 \mathrm{e}-4(4.2)$ & $1.21 \mathrm{e}-4(3.9)$ \\
\hline $.662 \mathrm{e}-2$ & 216 & $6.52 \mathrm{e}-5(5.6)$ & $3.65 \mathrm{e}-5(4.2)$ & $2.98 \mathrm{e}-5(4.1)$ \\
\hline $.331 \mathrm{e}-2$ & 432 & $1.40 \mathrm{e}-5(4.7)$ & $8.85 \mathrm{e}-6(4.1)$ & $7.86 \mathrm{e}-6(3.8)$ \\
\hline $.166 \mathrm{e}-2$ & 864 & $2.68 \mathrm{e}-6(5.2)$ & $2.15 \mathrm{e}-6(4.1)$ & $2.04 \mathrm{e}-6(3.9)$ \\
\hline \hline
\end{tabular}

(a) $L_{1}$ volume errors.

\begin{tabular}{|l|c|l|l|l|}
\hline$h$ & $N_{x}, N_{y}$ & 1st order grad. & ptwise grad. & 2nd order grad. \\
\hline .5297 & 27 & $6.84 \mathrm{e}-02$ & $3.31 \mathrm{e}-02$ & $2.37 \mathrm{e}-2$ \\
\hline .2648 & 54 & $2.83 \mathrm{e}-02(2.4)$ & $1.21 \mathrm{e}-02(2.7)$ & $8.183-3(2.9)$ \\
\hline .1324 & 108 & $1.03 \mathrm{e}-02(2.8)$ & $4.69 \mathrm{e}-03(2.6)$ & $3.453-3(2.4)$ \\
\hline $.662 \mathrm{e}-2$ & 216 & $3.65 \mathrm{e}-03(2.8)$ & $1.82 \mathrm{e}-03(2.6)$ & $1.38 \mathrm{e}-3(2.5)$ \\
\hline $.331 \mathrm{e}-2$ & 432 & $1.24 \mathrm{e}-03(3.0)$ & $7.18 \mathrm{e}-04(2.6)$ & $6.15 \mathrm{e}-4(2.2)$ \\
\hline $.166 \mathrm{e}-2$ & 864 & $3.96 \mathrm{e}-04(3.1)$ & $2.85 \mathrm{e}-04(2.6)$ & $2.58 \mathrm{e}-4(2.4)$ \\
\hline \hline
\end{tabular}

(b) $L_{1}$ boundary errors.

Table 1: L1 norm of the error in the domain volume and along the boundary, for the supersonic vortex example. The error using first order accurate gradients, pointwise quadratic, and fully second order accurate gradients is shown. Pointwise quadratic gradients have half of the error than first order gradients. Truly second order accurate gradients are even better, especially on coarser grids. The convergence rate is given in parentheses.

the same behavior using local time stepping, which does not need SRD stabilization. It has the same convergence rate, showing that this decrease is not due to SRD.

Figure 9 and Table 1 show that using pointwise quadratic cut cell gradients is roughly a factor of 2 more accurate on coarser grids. The more complicated second order accurate gradient is even more accurate, especially on coarser grids. Ultimately the gradient error is reduced, and the error curves for the first and second order accurate gradients approach each other.

We also use this example to compare the effect of state redistribution versus marching to steady state using local time-stepping without SRD. Table 2 shows a comparison of the error in the converged solution using local time stepping (LTS) without SRD, and the error with full time steps and SRD stabilization, both using first order accurate 


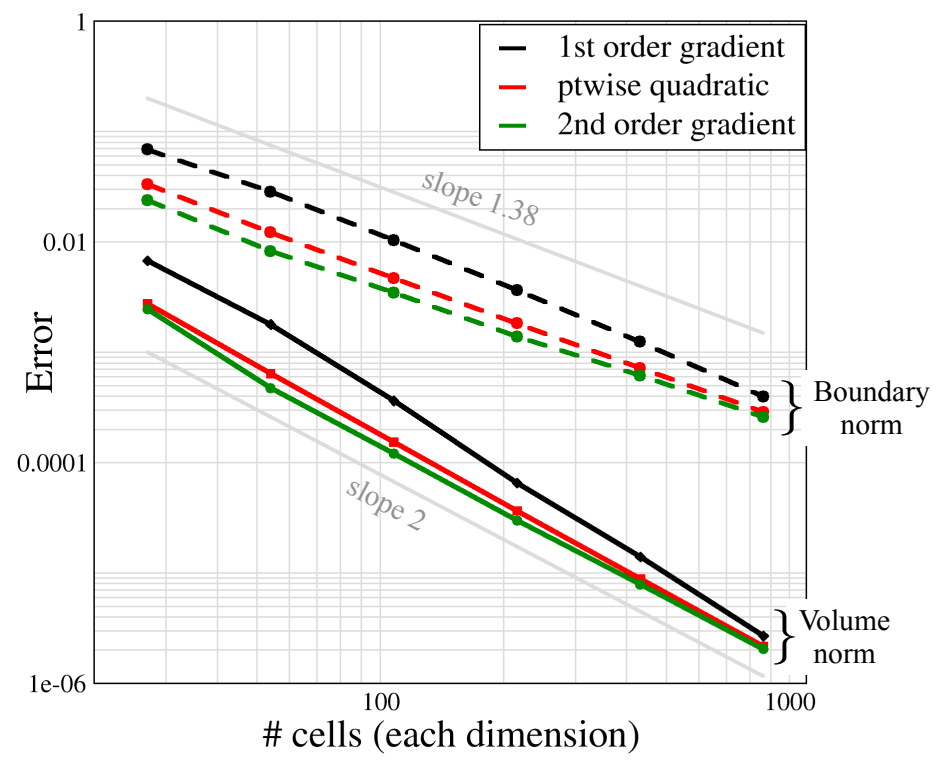

Figure 9: Convergence in the L1 norm of the error in the entire domain (solid line), and along the boundary (dashed line). The reference line has slope 2 next to the domain error. The convergence rate at the boundary is between 1.38 and 1.5 along the boundary.

gradients. The convergence rates and errors are essentially identical, showing that SRD does not degrade the computed solution with too much diffusion due to the merging neighborhoods. This holds across all the other gradient formulations too.

\subsection{Shock Reflection from Cylinder}

Next we demonstrate the method using a Mach 2 shock diffracting around a circular cylinder.

The shock will meet the cylinder and reflect at the cut cells, which are at all angles in the mesh. This example will demonstrate the robustness of SRD, and demonstrate the smoothness of the density profile around the cylinder, despite the completely irregular mesh.

A cylinder with radius 0.15 is centered at $(0.5,0.5)$, and the shock is initially located at $x=0.2$. For this example we compare results using the MUSCL scheme and the Method of Lines as the base schemes, both using local Lax Friedrichs for the Riemann solver. Both methods use second order accurate gradients. The BJ limiter is used to 


\begin{tabular}{|l|c|l|l|l|l|}
\hline$h$ & $N_{x}, N_{y}$ & \multicolumn{2}{|c|}{ Volume Error } & \multicolumn{2}{c|}{ Boundary Error } \\
\hline & & LTS (no SRD) & SRD & LTS (no SRD) & SRD \\
\hline .5297 & 27 & $6.09 \mathrm{e}-3$ & $6.75 \mathrm{e}-3$ & $6.75 \mathrm{e}-02$ & $6.84 \mathrm{e}-02$ \\
\hline .2648 & 54 & $1.67 \mathrm{e}-3(3.6)$ & $1.78 \mathrm{e}-4(3.8)$ & $2.79 \mathrm{e}-02(2.4)$ & $2.83 \mathrm{e}-02(2.4)$ \\
\hline .1324 & 108 & $3.41 \mathrm{e}-3(4.9)$ & $3.63 \mathrm{e}-4(4.9)$ & $1.00 \mathrm{e}-02(2.8)$ & $1.03 \mathrm{e}-02(2.8)$ \\
\hline $.662 \mathrm{e}-2$ & 216 & $6.62 \mathrm{e}-5(5.2)$ & $6.52 \mathrm{e}-5(5.6)$ & $3.51 \mathrm{e}-03(2.9)$ & $3.65 \mathrm{e}-03(2.8)$ \\
\hline $.331 \mathrm{e}-2$ & 432 & $1.34 \mathrm{e}-5(4.9)$ & $1.40 \mathrm{e}-5(4.7)$ & $1.21 \mathrm{e}-03(2.9)$ & $1.24 \mathrm{e}-03(3.0)$ \\
\hline $.166 \mathrm{e}-2$ & 864 & $2.59 \mathrm{e}-6(5.2)$ & $2.68 \mathrm{e}-6(5.2)$ & $3.78 \mathrm{e}-04(3.2)$ & $3.96 \mathrm{e}-04(3.1)$ \\
\hline \hline
\end{tabular}

Table 2: Comparison of errors for supersonic vortex problem using local time stepping, which does not use SRD, and regular time stepping with SRD. The errors are very similar, showing that SRD does not degrade the solution with too much diffusion.

limit both the base scheme irregular cells and neighborhood reconstruction gradients. The CFL for the MUSCL scheme was 0.85, and for the MOL scheme was 1.0 using (22), based on the full cell volumes.

Figure 10 left shows the solution density from MUSCL, and right from MOL, at time $T=0.25$. Both grids use 302 cells in each direction, and the domain is $[0.0,1.00001$, by $[0.0,1.0]$, again to prevent mesh degeneracies. There are 416 cut cells around the cylinder; 160 of the cut cells had volume fractions less than 0.5 and were stabilized with SRD. The smallest volume fraction was 1.17e-4. For comparison, this is also the time shown in [13. Here and in [13, the solution in front of the cylinder where the maximum density is located, behaves better with the one-step methods than with MOL. The method of lines solution is smoother around the boundary than our MUSCL variant.

Figure 11 shows the density profile from both schemes taken along the cylinder. For this plot, the cut cell variable is reconstructed to the midpoint of the cylinder line segment in each cell. The zoom shows the difference more clearly. 

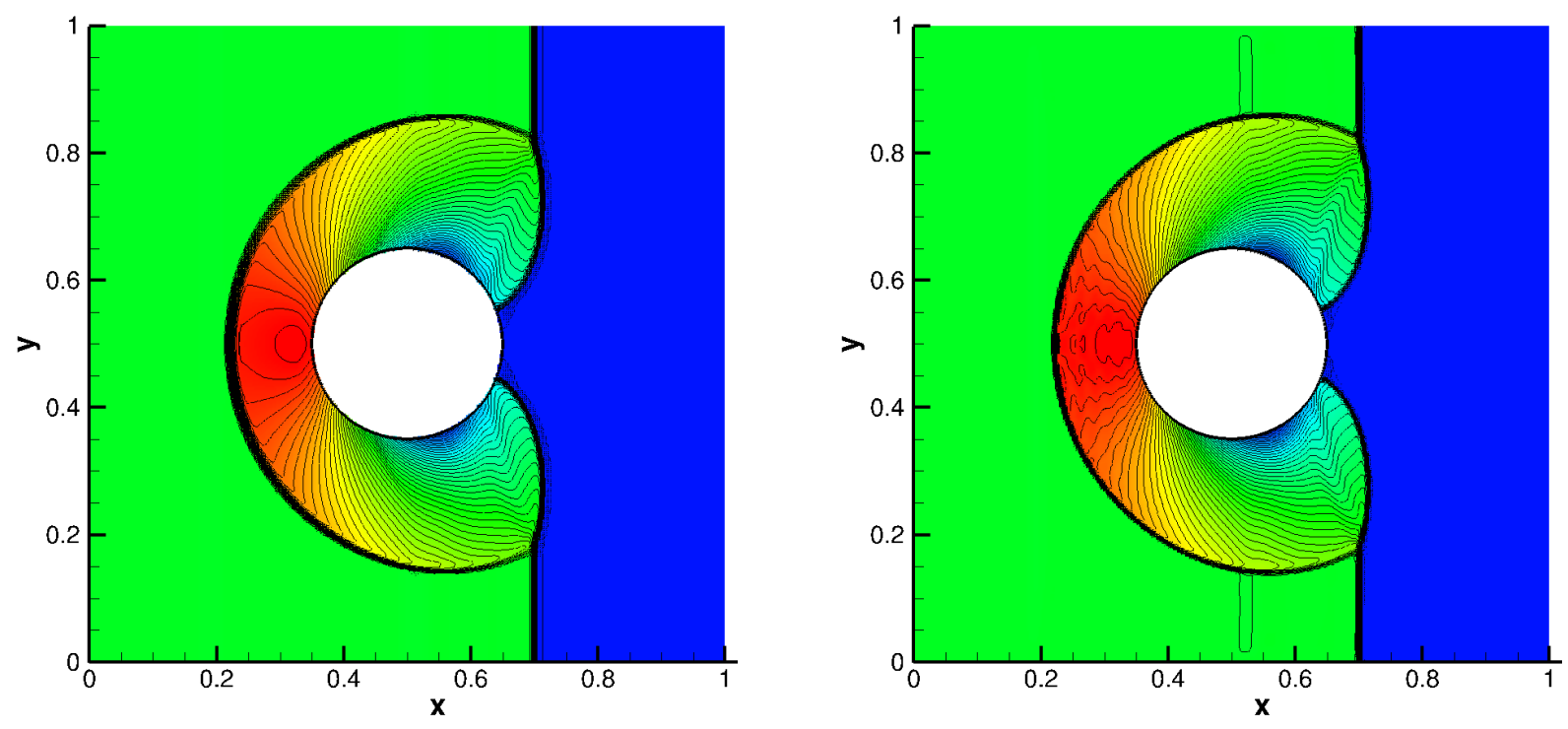

Figure 10: Density profile of Mach 2 shock reflection around a cylinder, at time $T=0.25$. Left computation uses MUSCL, right uses MOL. There are 52 contours between 1.3 and 6.5. Both schemes robustly compute the solution. The subsonic region in front of the cylinder is better behaved with MUSCL.
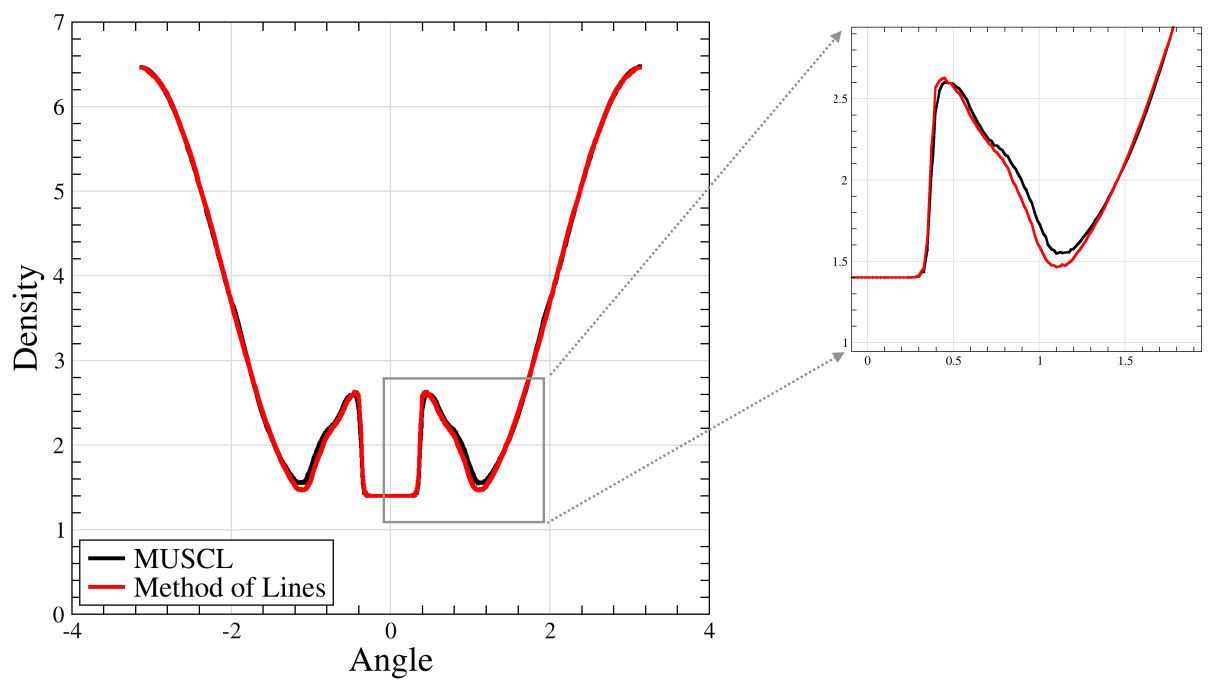

Figure 11: The density profile around the cylinder for the MUSCL and MOL schemes at time $T=0.25$. The methods give somewhat different profiles at the local minima, but the shock is located at the same position. Both methods are remarkably smooth considering the irregularity of the cut cell mesh. 


\subsection{Double Mach Reflection problem}

We present one final standard test case for embedded boundary meshes. We reflect a Mach 10 shock obliquely over a wedge, where the shock and wall form a $60^{\circ}$ angle. The problem domain is $[0,3.0] \times[0,1.75]$, with an angled wall passing through the point $(1 / 6,0)$. We use the MUSCL scheme, with the interior slopes computed using monotonized central differences. The irregular cells and SRD neighborhoods use second order accurate gradients, and limit using BJ. The CFL is 0.85 .
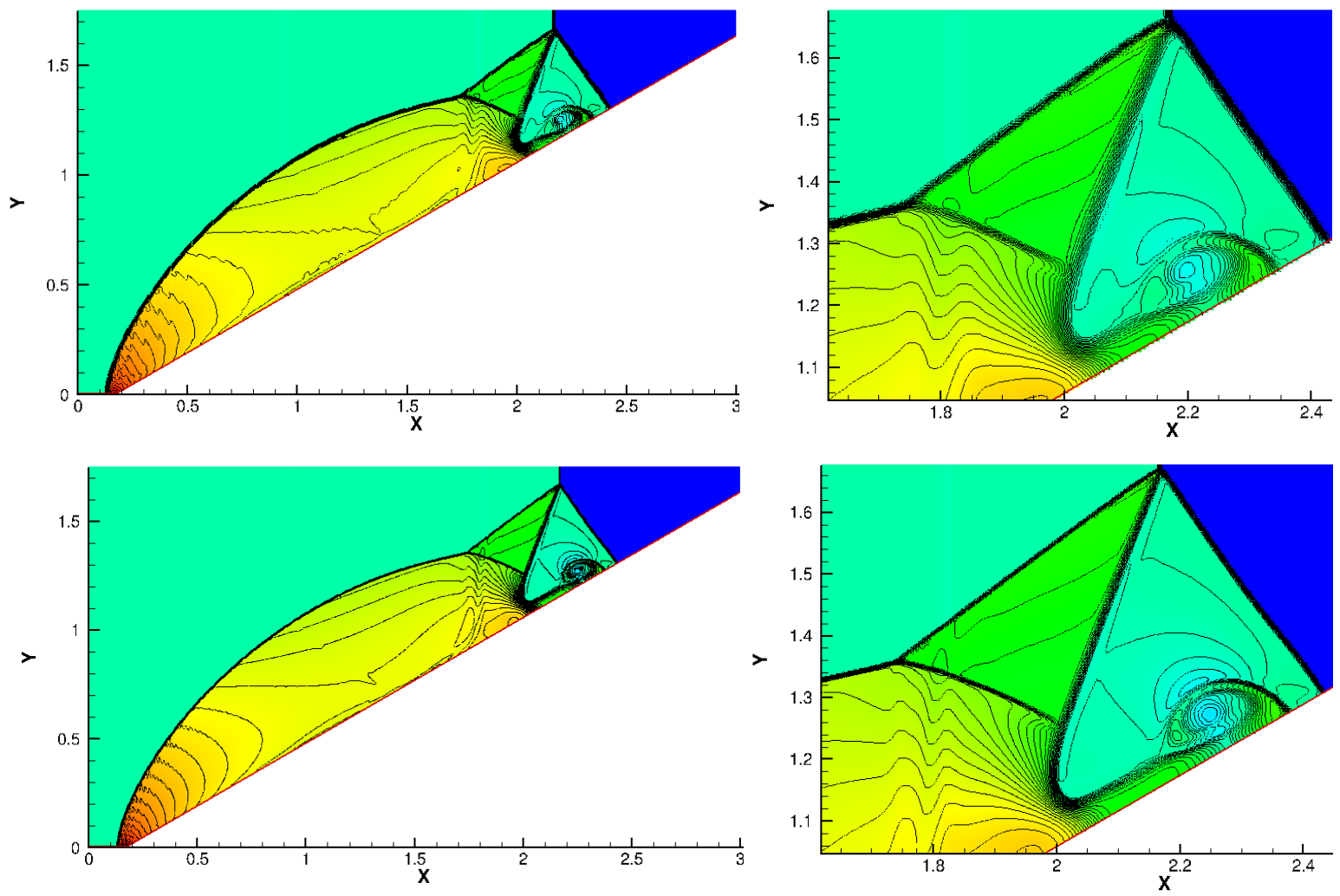

Figure 12: Density plot and isolines of a Mach 10 shock impinging obliquely on a wedge at time $T=0.2$ where $\Delta x=\Delta y=1 / 240$ (top) and $1 / 480$ (bottom). The solution on the full domain is shown adjacent to a zoom of the Mach stem region. Sixty isolines between 1.39 and 21.0 are drawn.

The solution to this problem is a complex, self-similar reflection pattern composed of incident and reflected shocks and contact discontinuities [24, 8]. The contact discontinuities are an unstable feature of the solution that can be difficult to resolve cor- 
rectly, especially in the neighborhood of the reflecting boundary where the carbuncle phenomenon can occur [16].

The solution at the final time $T=0.2$ is plotted in Figure 12, where the grid resolution is $\Delta x=\Delta y=1 / 240$. We obtain qualitatively comparable results to those in [8] on the same grid resolution. We also show for comparison the results using $\Delta x=\Delta y=1 / 480$, also done in [8]. Again, the improvement is very similar. Finally, in Figure 13 we show the density along the boundary for three resolutions. Despite the irregularity of the cut cells, the solution is very smooth. The smallest cut cell in the coarser grid has volume fraction 1.65e-6. On the finest grid the smallest volume fraction is $2.95 \mathrm{e}-7$.

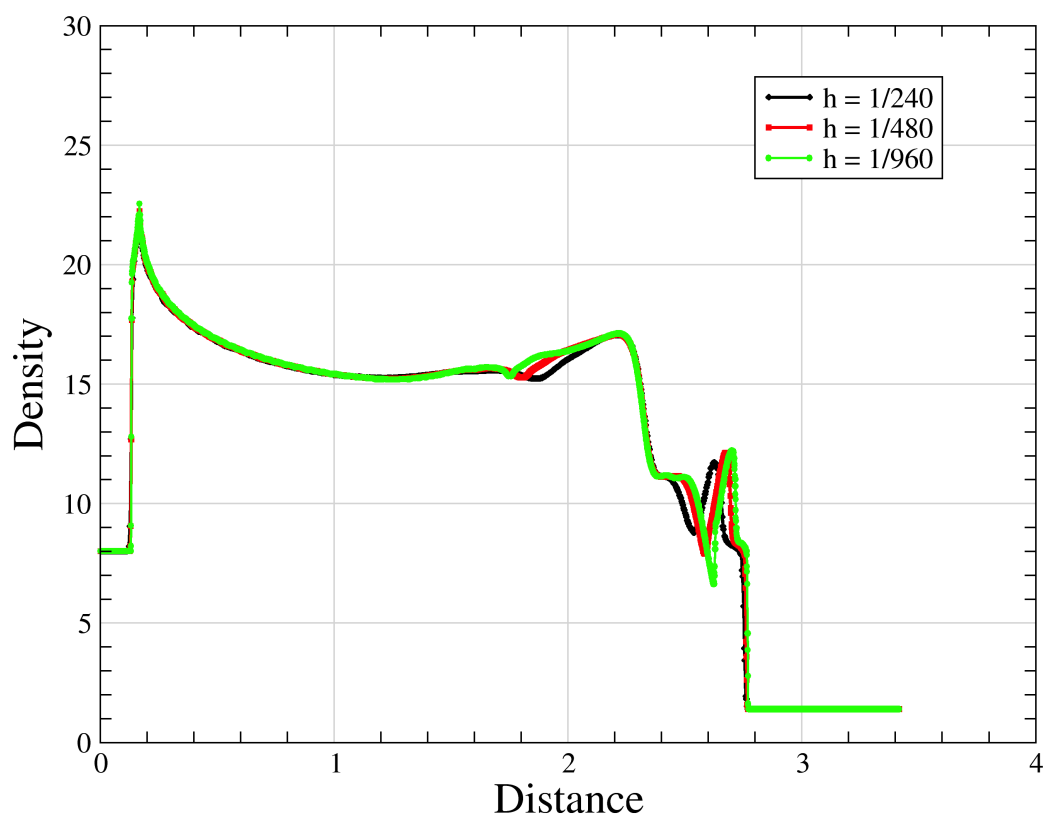

Figure 13: Density plotted along the wall, as a function of distance from the lower left corner of the domain. A finer run is included to show the trend. The shocks are in the same place on all grids, but the unstable contact is slightly shifted.

\section{Conclusions}

We have presented a state redistribution algorithm to solve the small cell problem on cut cell meshes. It is conservative, allows for overlapping temporary merging neighborhoods so is easy to implement, and is linearity preserving. Numerical experiments show that on 
smooth problems, second order accuracy is maintained, and the solution is not degraded by the postprocessing. For problems with shocks, the scheme maintains robustness at the cut cells. We have shown experiments using SRD on two different base schemes, but it should be applicable to any underlying numerical method with cell-centered variables.

It should be straightforward to apply state redistribution to three dimensional applications. It should also be applicable to different sets of equations, e.g. incompressible flow. It seems clear that when used in conjunction with a higher order base scheme, state redistribution can be extended to higher order accuracy. We have already started doing this for 3rd and 4th order accuracy. However, higher order methods bring in many new features, so we do not include that here.

\section{Acknowledgments}

We thank Michael Aftosmis, Sandra May, and Marian Nemec for carefully reading the manuscript and many helpful suggestions. This research was partially supported by the U.S. Department of Energy under contract DE-FG02-88ER25053.

\section{References}

[1] M. Aftosmis, D. Gaitonde, and T. S. Tavares. On the accuracy, stability and monotonicity of various reconstruction algorithms for unstructured meshes. AIAA-94-0415, Jan. 1994.

[2] T. Barth and D. Jespersen. The design and application of upwind schemes on unstructured meshes. AIAA-89-0366, 1989.

[3] M.J. Berger. A note on the stability of cut cells and cell merging. Applied Numerical Mathematics, 96:180-186, 2016.

[4] M.J. Berger and C. Helzel. A simplified $h$-box method for embedded boundary grids. SIAM J. Sci. Comput., 34:861-888, 2012.

[5] D. Cecere and E. Giacomazzi. An immersed volume method for large eddy simulation of compressible flows using a staggered-grid approach. Comput. Methods Appl. Mech. Engrg., 280:1-27, 2014.

[6] I. Chern and P. Colella. A conservative front tracking method for hyperbolic conservation laws. Technical Report UCRL-97200, Lawrence Livermore National Laboratory, July 1987.

[7] M.H. Chung. Cartesian cut cell approach for simulating incompressible flows with rigid bodies of arbitary shape. Comp. Fluids, 35:607-623, 2006. 
[8] B. Cockburn and C-W. Shu. The Runge-Kutta Discontinuous Galerkin method for conservation laws V: Multidimensional systems. J. Comp. Phys, 141(2):199 - 224, 1998.

[9] P. Colella. Multidimensional upwind methods for hyperbolic conservation laws. J. Comp. Physics, 87:171-200, 1990.

[10] P. Colella, D.T. Graves, B.J. Keen, and D. Modiano. A Cartesian grid embedded boundary method for hyperbolic conservation laws. J. Comp. Phys., 211:347-366, 2006.

[11] A. Giuliani and L. Krivodonova. Analysis of slope limiters on unstructured triangular meshes. J. Comp. Phys., 374:1-26, 2018.

[12] N. Gokhale, N. Nikiforakis, and R. Klein. A dimensionally split Cartesian cut cell method for hyperbolic conservation laws. J. Comp. Phys., 364:186-208, July 2018.

[13] C. Helzel, M. J. Berger, and R. J. LeVeque. A high-resolution rotated grid method for conservation laws with embedded geometries. SIAM J. Sci. Comput., 26:785-809, 2005.

[14] X.Y. Hu, B.C. Khoo, N.A. Adams, and F.L. Huang. A conservative interface method for compressible flows. J. Comp. Phys., 219:553-578, 2006.

[15] D.M. Ingram, D.M. Causon, and C.G. Mingham. Developments in Cartesian cut cell methods. Mathematics and Computers in Simulation, 61:561-572, 2003.

[16] F. Kemm. Heuristical and numerical considerations for the carbuncle phenomenon. Appl. Math. Comp., 320:596 - 613, 2018.

[17] M.P. Kirkpatrick, S.W. Armfield, and J.H. Kent. A representation of curved boundaries for the solution of the Navier-Stokes equations on a staggered three-dimensional Cartesian grid. J. Comp. Phys., 184:1-36, 2003.

[18] L. Krivodonova and M. Berger. High-order accurate implementation of solid wall boundary conditions in curved geometries. J. Comp. Phys., 221:492-512, 2006.

[19] S. May and M.J. Berger. An explicit implicit scheme for cut cells in embedded boundary meshes. J. Sci. Comput., 2016. doi=10.1007/s10915-016-0326-2.

[20] B. Muralidhara and S. Menon. A high-order adaptive Cartesian cut-cell method for simulation of compressible viscous flow over immersed bodies. J. Comp. Phys., 321:342$368,2016$.

[21] M. Nemec and M.J. Aftosmis. Toward automatic verification of goal-oriented flow simulations. Tech. Memo NASA/TM-2014-218386, National Aeronautics and Space Administration, 2014.

[22] B. van Leer. Toward the ultimate conservative difference scheme V. A second order sequel to Godunov method. J. Comp. Phys., 32:101-136, 1979. 
[23] B. Wendroff and A.B. White. A supraconvergent scheme for nonlinear hyperbolic systems. Comput. Math. Appl., 18, 1989.

[24] P. Woodward and P. Colella. The numerical simulation of two-dimensional fluid flow with strong shocks. J. Comp. Phys., 54(1):115 - 173, 1984. 